\title{
EVIDENCE FOR A TRANSITION REGION RESPONSE TO PENUMBRAL MICROJETS IN SUNSPOTS
}

\author{
G. J. M. Vissers, L. H. M. Rouppe van der Voort, and M. Carlsson \\ Institute of Theoretical Astrophysics, University of Oslo, P.O. Box 1029 Blindern, NO-0315 Oslo, Norway; g.j.m.vissers@astro.uio.no \\ Received 2015 August 11; accepted 2015 September 3; published 2015 September 29
}

\begin{abstract}
Penumbral microjets (PMJs) are short-lived, fine-structured, and bright jets that are generally observed in chromospheric imaging of the penumbra of sunspots. Here we investigate their potential transition region signature by combining observations with the Swedish 1-m Solar Telescope in the Ca II H and Ca II 8542 A lines with ultraviolet imaging and spectroscopy obtained with the Interface Region Imaging Spectrograph (IRIS), which includes the C II $1334 / 1335 \AA$, Si IV 1394/1403 $\AA$, and Mg II h \& k 2803/2796 ^lines. We find a clear corresponding signal in the IRIS Mg II k, C II, and Si IV slit-jaw images, typically offset spatially from the Ca II signature in the direction along the jets: from base to top, the PMJs are predominantly visible in Ca II, $\mathrm{Mg}$ II $\mathrm{k}$, and $\mathrm{C}$ II/Si IV, suggesting progressive heating to transition region temperatures along the jet extent. Hence, these results support the suggestion from earlier studies that PMJs may heat to transition region temperatures.
\end{abstract}

Key words: sunspots - Sun: activity - Sun: chromosphere - Sun: transition region

\section{INTRODUCTION}

Penumbral microjets (PMJs) are a prime example of the finestructured dynamics observed in active regions and in sunspots in particular. They were first reported by Katsukawa et al. (2007) in Ca II H imaging of a sunspot observed at different viewing angles with the Solar Optical Telescope (Tsuneta et al. 2008) on board Hinode (Kosugi et al. 2007) and were found to occur near bright penumbral grains. In regular imaging, but especially in time-difference movies, they pop up unmistakably as small-scale, short-lived jets with average lengths between 1 and $4 \mathrm{Mm}$, widths of a few hundred kilometers, lifetimes of up to a minute, and apparent extension speeds well over $100 \mathrm{~km} \mathrm{~s}^{-1}$. PMJs have also been observed in the wings of the Ca II $8542 \AA$ line, with a typically blue-overred asymmetric spectral profile peaking between 10 and $20 \mathrm{~km} \mathrm{~s}^{-1}$ blueward of the line core (Reardon et al. 2013; Drews 2014).

When observed at different viewing angles, their orientation with respect to the background penumbra is also revealing: toward the limb, microjets appear at an angle to the photospheric penumbral filaments, while they are well-aligned closer to disk center. This suggests that PMJs are (at least initially) more upright than the photospheric penumbral filaments and indeed inclination angles to the normal ranging between $20^{\circ}$ and $70^{\circ}$ have been found (Katsukawa et al. 2007), with a tendency for increasing angles from the inner to the outer penumbral boundary (Jurčák \& Katsukawa 2008). This outward trend is indicative of PMJs following the magnetic field as it expands from the initially near-vertical orientation in the photosphere to a more horizontal orientation in the chromosphere.

These observations also fit with a scenario in which the PMJs are driven by reconnection between penumbral magnetic fields. The darker penumbral filaments are known to host more horizontal fields, while the brighter penumbral areas and grains harbor more vertical fields (Langhans et al. 2005). As the latter move inward toward the sunspot umbra, the shearing reconnection between inclined penumbral fields may provide the necessary energy to drive the PMJs. Katsukawa \& Jurčák (2010) and Jurčák \& Katsukawa (2010) found downflows in the penumbra that in some cases appeared to be related to PMJs, fitting the reconnection scenario.

The launch of the Interface Region Imaging Spectrograph (IRIS; De Pontieu et al. 2014) has opened a new, highresolution window on the chromosphere and transition region and its data are therefore perfectly suited for investigating the potential transition region response to PMJs (the presence of which was already speculated by Katsukawa et al. 2007). By combining IRIS observations with data from the Swedish 1-m Solar Telescope (SST; Scharmer et al. 2003) for PMJ detection, this work aims at investigating the characteristic ultraviolet signature of the jets and the time-dependence of their visibility in chromospheric and transition region diagnostics.

\section{OBSERVATIONS AND ANALYSIS}

\subsection{Data Acquisition and Reduction}

For this study we analyzed two data sets of coordinated SST and IRIS observations obtained on 2014 September 5 and 6, tracking the leading sunspot in AR 12152. Figure 1 shows sample field-of-view images for both days in different SST and IRIS diagnostics. The CRisp Imaging SpectroPolarimeter (CRISP; Scharmer et al. 2008) at the SST recorded imaging spectroscopy in the $\mathrm{H} \alpha$ and $\mathrm{Ca}$ II $8542 \AA$ lines in addition to full Stokes polarimetry at one wavelength position in the blue wing of $\mathrm{Fe}_{\mathrm{I}} 6301 \AA$. The overall repeat cadence of this program is 11.6 s. CRISP observed from 07:58 to 09:52 UT and from 08:23 to 10:24 UT on the respective days. In addition, part of the light from the telescope was diverted before entering CRISP to provide $\mathrm{Ca}$ II $\mathrm{H}$ core (filter FWHM of $1.1 \AA$ ) and wide-band imaging. On September 5, the $\mathrm{Ca}$ II $\mathrm{H}$ data were obtained only during 07:59-09:07 UT, while on September 6 the full CRISP sequence was supported by $\mathrm{Ca}$ II $\mathrm{H}$ imaging. In this study, only Ca II $8542 \AA$ and $\mathrm{Ca}$ II $\mathrm{H}$ core data were used for further analysis.

During these coordinated observations, IRIS ran a four-step sparse raster (OBSID 3820255167) with $2 \mathrm{~s}$ exposures, wavelength binning by two in the far-ultraviolet (affecting only the spectra between 1332 and $1407 \AA$ ), and including context slit-jaw imaging in three channels, C II $1330 \AA$, Si IV $1400 \AA$, and $\mathrm{Mg}_{\text {II }} \mathrm{k} 2796 \AA$. The overall repeat cadence of 


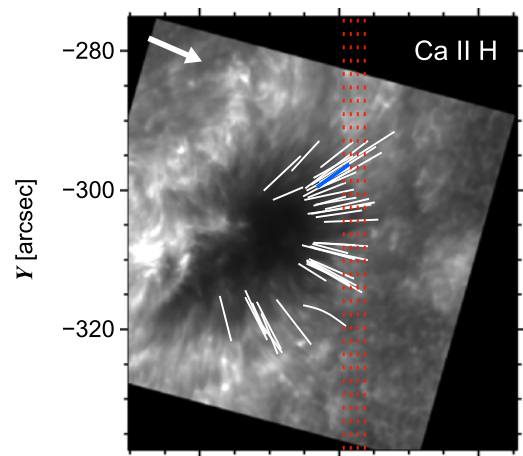

680

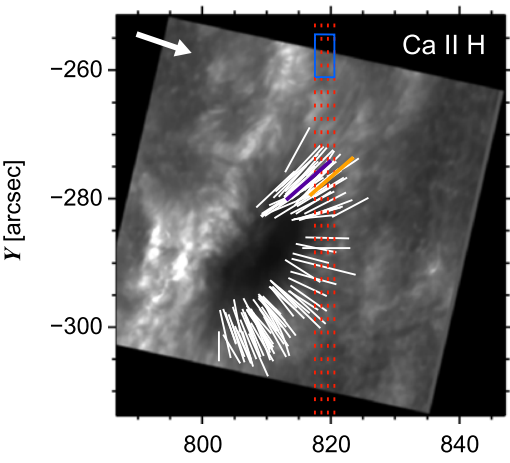

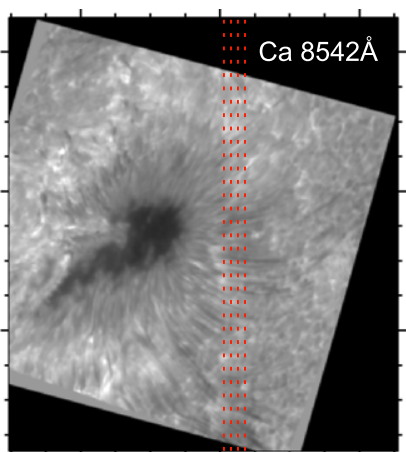

680

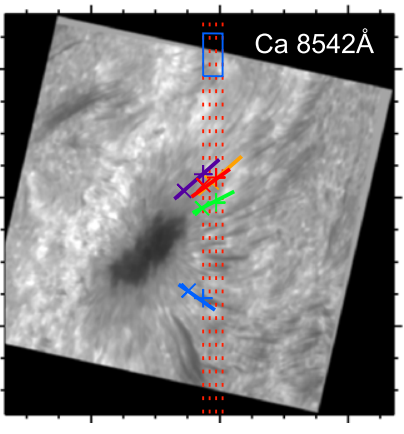

800

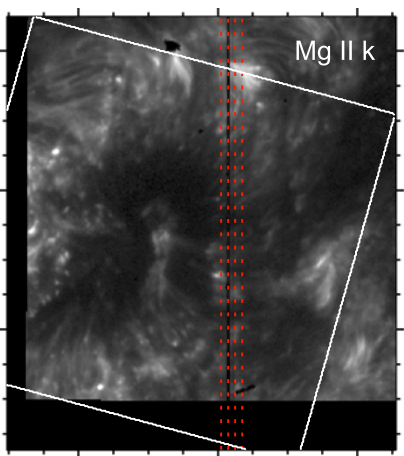

700

680

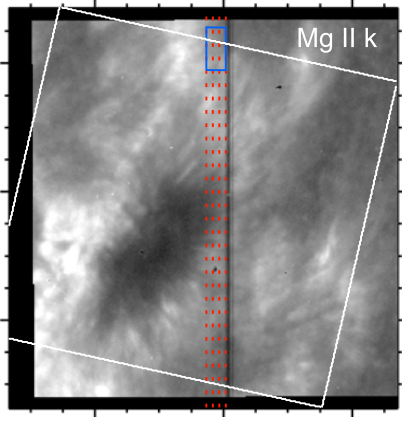

800

820

\begin{abstract}
840
\end{abstract}

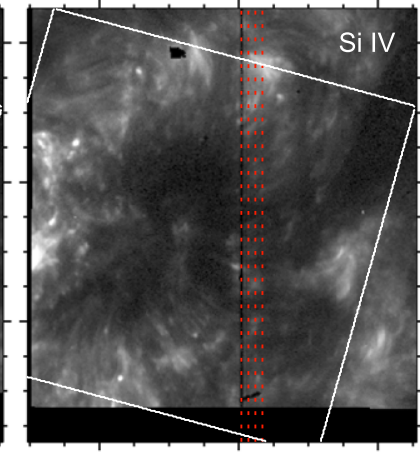

680

700

720

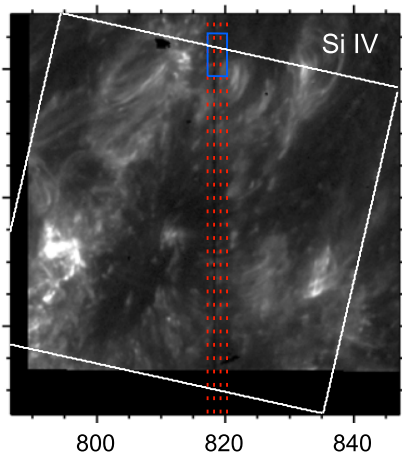

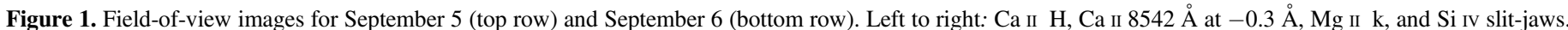

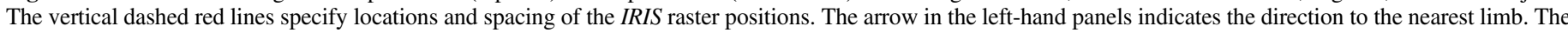

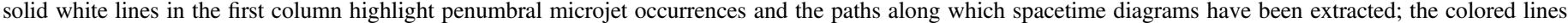

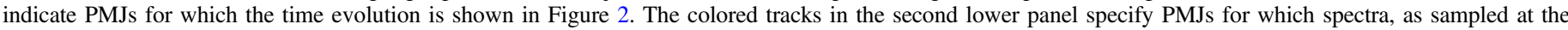
markers, are shown in Figure 5. The blue box in the lower panels specifies the averaging region for the mean spectrum in that same figure.

both the raster and the slit-jaws is $13 \mathrm{~s}$. IRIS co-observed between 08:06-11:06 UT and 08:05-11:01 UT on the respective days.

We used the CRISPRED pipeline (see de la Cruz Rodríguez et al. 2015 and references therein) for reduction of the SST/ CRISP data. The $\mathrm{Ca}$ II $\mathrm{H}$ data were processed using a similar set of procedures, including dark and flat-field corrections, as well minimization of high-order seeing effects using MultiObject Multi-Frame Blind Deconvolution (van Noort et al. 2005) and removal of rubber-sheet distortions through destretching following Shine et al. (1994). The resulting Ca II $\mathrm{H}$ data have the same $11.6 \mathrm{~s}$ cadence as the CRISP data. Coalignment was achieved using the procedures as described in Vissers et al. (2015) and using SST Ca II $8542 \AA / C a$ II $\mathrm{H}$ (for September 5 and 6, respectively) and IRIS Mg II k $2796 \AA$ as a reference. The CRisp SPectral EXplorer (CRISPEX; Vissers \& Rouppe van der Voort 2012) was extensively used during alignment and for subsequent data analysis.

\subsection{Event Selection}

PMJs were initially detected based on morphology and lifetime dynamics through visual inspection of $\mathrm{Ca}$ II $\mathrm{H}$ timedifference sequences constructed with a temporal offset of three time steps (about $35 \mathrm{~s}$ ). This follows the methodology that has been employed in several PMJ studies before (e.g., Katsukawa et al. 2007; Jurčák \& Katsukawa 2008). These identifications were subsequently checked against the regular $\mathrm{Ca}$ II $\mathrm{H}$ imaging to confirm their PMJ nature. We restricted our event sample for final analysis to occurrences when IRIS was not affected by increased cosmic ray hits (resulting from the orbital pass through the South Atlantic Anomaly), yielding a grand total of 180 events (along 147 paths, i.e., counting recurring jets separately), of which 52 were on September 5 and 128 on September 6. This selection of PMJs is highlighted by solid lines in the first column of Figure 1.

\section{RESULTS}

\subsection{Morphology and Time Evolution}

Figure 2 shows the time evolution of three example PMJs that are highlighted by colored tracks in the first column of Figure 1. Their visibility in different diagnostics is indicated through color-coding: $\mathrm{Ca}$ II $\mathrm{H}$ signal is red, $\mathrm{Mg}$ II $\mathrm{k}$ is green, and $\mathrm{Si}$ IV or $\mathrm{C}$ II is blue. For events that are visible in multiple channels the colors add: a feature visible in $\mathrm{Ca}$ II $\mathrm{H}$ and $\mathrm{Mg}$ II $\mathrm{k}$ turns yellow, in $\mathrm{Mg}_{\text {II }} \mathrm{k}$ and $\mathrm{Si}$ IV more cyan, while something visible in all three will be white. Using nearest-neighbor selection, the time differences between the diagnostics are small, but non-zero nevertheless: about $3.3 \mathrm{~s}$ on average and $6.7 \mathrm{~s}$ at most between the IRIS slit-jaws and SST, and 6.4 and $9.8 \mathrm{~s}$ between the $\mathrm{Mg}$ II $\mathrm{k}-\mathrm{Si}$ IV and $\mathrm{Mg}$ II $\mathrm{k}-\mathrm{C}_{\text {II }}$ pairs, respectively.

Although their lifetimes, sizes, and brightnesses differ, these examples display a generally observed rainbow-colored signature. The signal in $\mathrm{Ca}$ II $\mathrm{H}$ (or Ca II $8542 \AA$, not shown here) is predominantly found toward the base of the PMJ, $\mathrm{Mg}$ II $\mathrm{k}$ is observed throughout but mostly in the middle, while Si IV and $\mathrm{C}_{\mathrm{II}}$ are generally only observed toward the PMJ tops. While the $\mathrm{Ca}$ II and $\mathrm{Mg}$ II $\mathrm{k}$ signal are elongated and jet-like in 


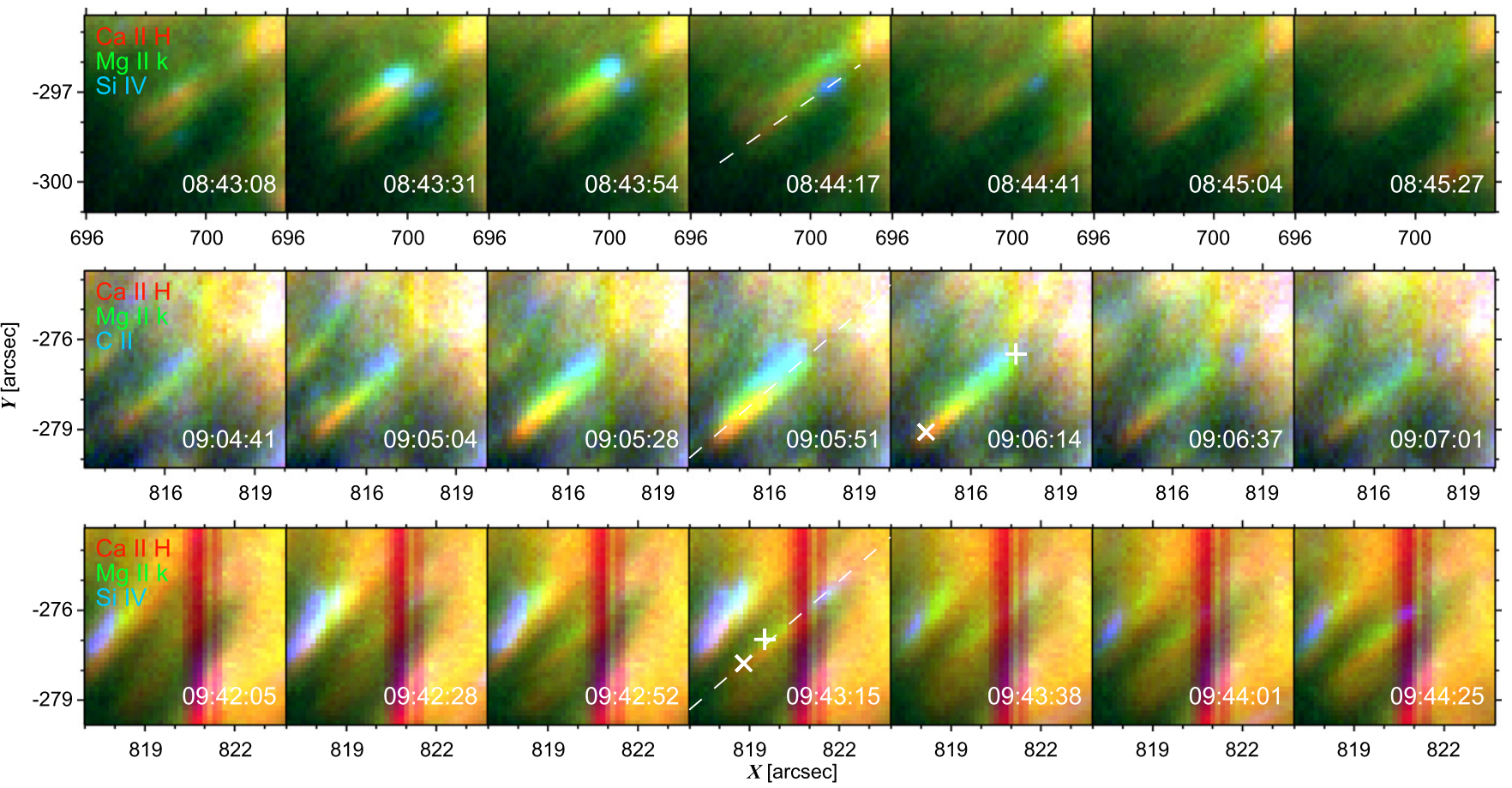

Figure 2. Time evolution of three PMJ examples. From top to bottom these correspond to the blue, violet, and orange tracks highlighted in the left-hand column of Figure 1. The diagnostic color-coding is indicated in the top left of each panel, while the time in UT is specified at the bottom. The dashed white lines specify the paths along which spacetime diagrams (b), (f), and (h) (shown in Figure 3) have been extracted, while the symbols correspond to sampling locations and timing of the violet and orange Ca II $8542 \AA$ (cross marker, $\times$ ) and IRIS (plus marker, +) spectra shown in Figure 5. Note that the bright event to the left in the bottom example resulted from multiple fine-structure loops brightening (distinguishable only at SST resolution) and exhibited "IRIS bomb"-type (Peter et al. 2014) UV profiles, in contrast to all PMJ spectra analyzed; it was therefore discarded as a microjet in our analysis.
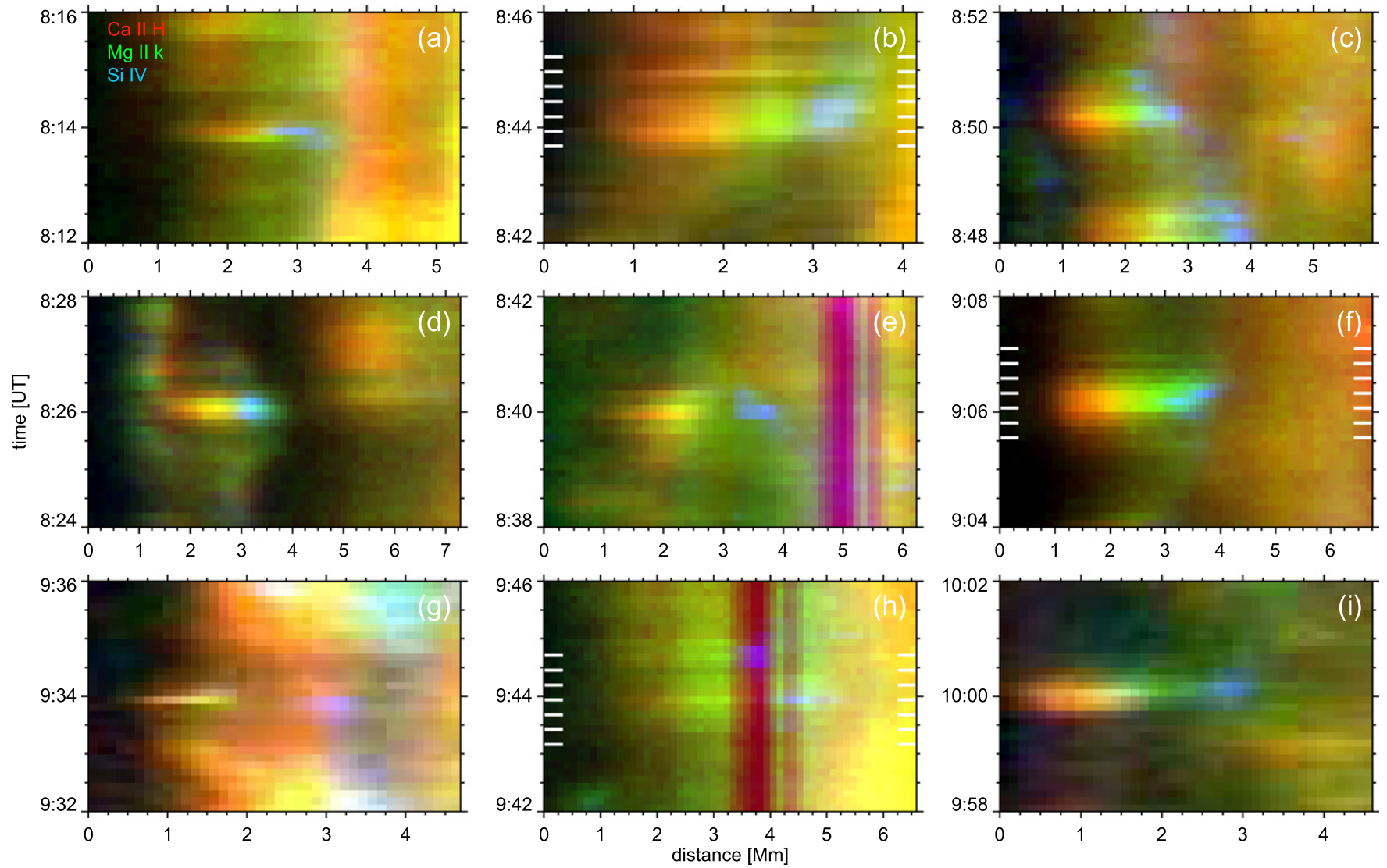

Figure 3. Spacetime diagrams for nine representative PMJs on September 5, panels (a)-(c), and September 6, panels (d)-(i). The color-coding convention is as for Figure 2. The thick white inner tickmarks in panels (b), (f), and (h) correspond to the times for which Figure 2 shows cutout intensity images. 

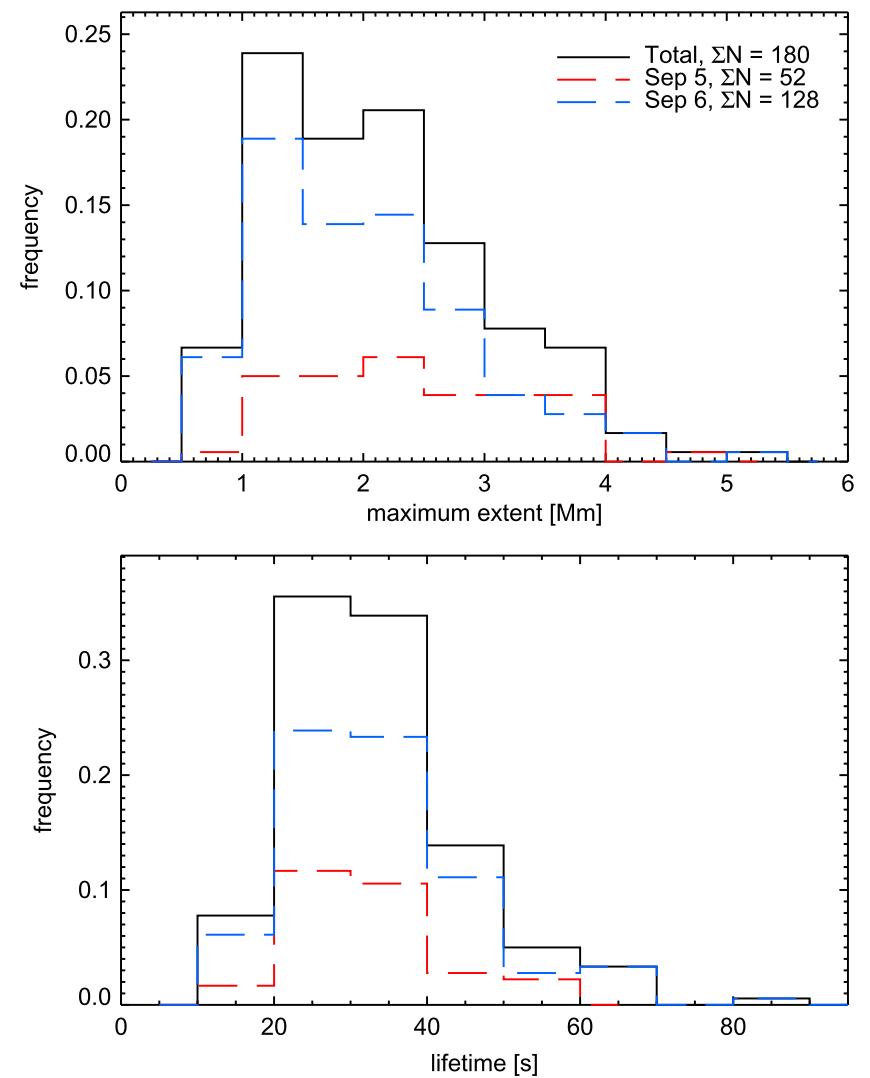

Figure 4. Distribution of maximum PMJ extent (top) and visibility lifetime (bottom) as determined from the spacetime diagrams of all PMJs shown in Figure 1. Different line styles indicate different samples, as indicated in the top right of the top panel.

shape, the Si IV and $\mathrm{C}$ II signature is more often roundish. Given the viewing angle, this is unlikely an effect of foreshortening, as the jets are viewed from the side. The $\mathrm{C}_{\text {II }}$ PMJ signal appears somewhat more elongated than $\mathrm{Si}$ IV and is sometimes also offset spatially; for some events this may be an intrinsic effect as the offset is in the direction perpendicular to the extension of the jet, but for most cases it seems likely a result of the non-negligible $3.5 \mathrm{~s}$ time delay between the $\mathrm{C}_{\mathrm{II}}$ and Si IV exposures.

Spacetime diagrams were extracted along all paths specified in Figure 1 in order to further analyze the PMJ time evolution. Figure 3 presents a representative subset of those. The rainbowcolored pattern is clearly visible in all examples, but the details differ from case to case. For instance, in panel (a) the $\mathrm{Mg}$ II $\mathrm{k}$ signal appears to be strongly present prior to the visibility in $\mathrm{Ca}$ II $\mathrm{H}$ and $\mathrm{Si}$ IV, while in all other panels the $\mathrm{Mg}$ II $\mathrm{k}$ and $\mathrm{Ca}$ II $\mathrm{H}$ signal is roughly co-temporal. Indeed, in most panels the PMJ appears at the same time or within one time step in all three diagnostics. Panels (b) and (c) suggest jet recurrence on the order of 1-2 minutes along the same path and the latter panel also evidences the radial inward migration of PMJs that was already shown by Katsukawa et al. (2007). The Si IV signal in panels (c) and (e) appears to retract during the PMJ lifetime (with apparent speeds of about $2-4 \mathrm{~km} \mathrm{~s}^{-1}$ ), while the opposite apparent motion (at about $10 \mathrm{~km} \mathrm{~s}^{-1}$ ) is clearly visible in, for instance, panel (f). Some PMJs are only visible for 10-20 s (e.g., panels (a), (g), and (h)), while others remain visible for up to a minute (e.g., panels (e) and (f)).
Figure 4 visualizes these properties statistically, showing the frequency distribution of maximum extent and lifetimes. Both represent the combined visibility in $\mathrm{Ca}$ II $\mathrm{H}, \mathrm{Mg}$ II $\mathrm{k}$, and $\mathrm{Si}$ IV, which especially in terms of spatial extent is appreciably larger than for $\mathrm{Ca}$ II $\mathrm{H}$ alone (as evident from Figure 3). We find typical lengths ranging between about 1 to $5 \mathrm{Mm}$ for PMJs from both data sets, with an overall average of $2.1 \mathrm{Mm}$, however, nearly $57 \%$ of the PMJs are shorter than that. The lifetime distribution is equally skewed to lower values, ranging between 10 and $90 \mathrm{~s}$ and with an average of slightly over half a minute. Close to $78 \%$ of the PMJs are visible for $35 \mathrm{~s}$ or less.

\subsection{Spectral Signature}

Although many of the traced paths in Figure 1 are partially covered by the IRIS raster, the number of events for which spectra are available is limited (note that the traced paths indicate the track along which spacetime diagrams have been obtained, not the actual extent of the PMJs). Not only do not all PMJs along those tracks reach the leftmost raster position, but of those that do, the $\mathrm{Mg}$ II, Si IV, or C II slit-jaw signal is not always strong, resulting in an only weakly enhanced spectral signal. For September 5, 10 events show a response in either $\mathrm{C}$ II $/ \mathrm{Si}$ IV or $\mathrm{Mg}$ II $\mathrm{h} \& \mathrm{k}$, and only one is enhanced in all lines; for September 6, 12 events show response in either of the lines and 7 are enhanced in all of them. Nonetheless, the IRIS spectra show that the visibility of PMJs in the $\mathrm{Mg}_{\text {II }} \mathrm{k}$, Si IV, and C II slit-jaw images is a result of an enhancement of the spectral lines dominating their passbands.

Figure 5 shows sample spectra for five PMJs from September 6 with the strongest IRIS response and which are highlighted with the same color-coding in the second lower panel of Figure 1. In addition, the violet and orange curves correspond respectively to the PMJs shown in the middle and lower panels of Figure 2, and the (f) and (h) spacetime diagrams in Figure 3.

For all PMJs, the Ca II $8542 \AA$ line shows the typical blue-overred wing asymmetry, peaking out at $10-15 \mathrm{~km} \mathrm{~s}^{-1}$ blueward of the nominal line center. The red wing is generally smooth with an intensity close to the field-of-view average, but the blue and violet samplings also show a red wing enhancement at similar Doppler shift, albeit still lower in intensity than the blue wing peak.

Excluding the orange sampling (which was chosen to show the $\mathrm{Mg}$ II $\mathrm{h} \& \mathrm{k}$ response), the $\mathrm{Si}$ IV lines appear the most enhanced of the IRIS diagnostics. The peak ratio of the Si IV 1394 to $1403 \AA$ lines varies between 1.8 and 2.0, close enough to their transition probability of 2 to suggest optically thin formation for all samplings shown. The behavior of both $\mathrm{Si}$ IV lines is also similar: both profiles rise by a factor of 3-5 with respect to the quiescent profiles, while broadening appreciably (up to almost a factor of two for the broadest red profiles). With the exception of the blue and green profiles, which show blueshifts of about $10 \mathrm{~km} \mathrm{~s}^{-1}$ with respect to the average, the Si Iv lines are generally symmetric and show close to zero Doppler shift. There is no sign of O IV 1399.78 or $1401.16 \AA$ signal (labeled in the top right panel) for any of the samplings.

By comparison, the $\mathrm{C}_{\text {II }}$ lines appear rather docile. Only the blue sampling shows considerable enhancement over the quiescent profile, while all other samplings are dominated by noise. The blue profiles show a hint of red asymmetry, which is not reflected by the Si IV lines, but which is visible in both the 


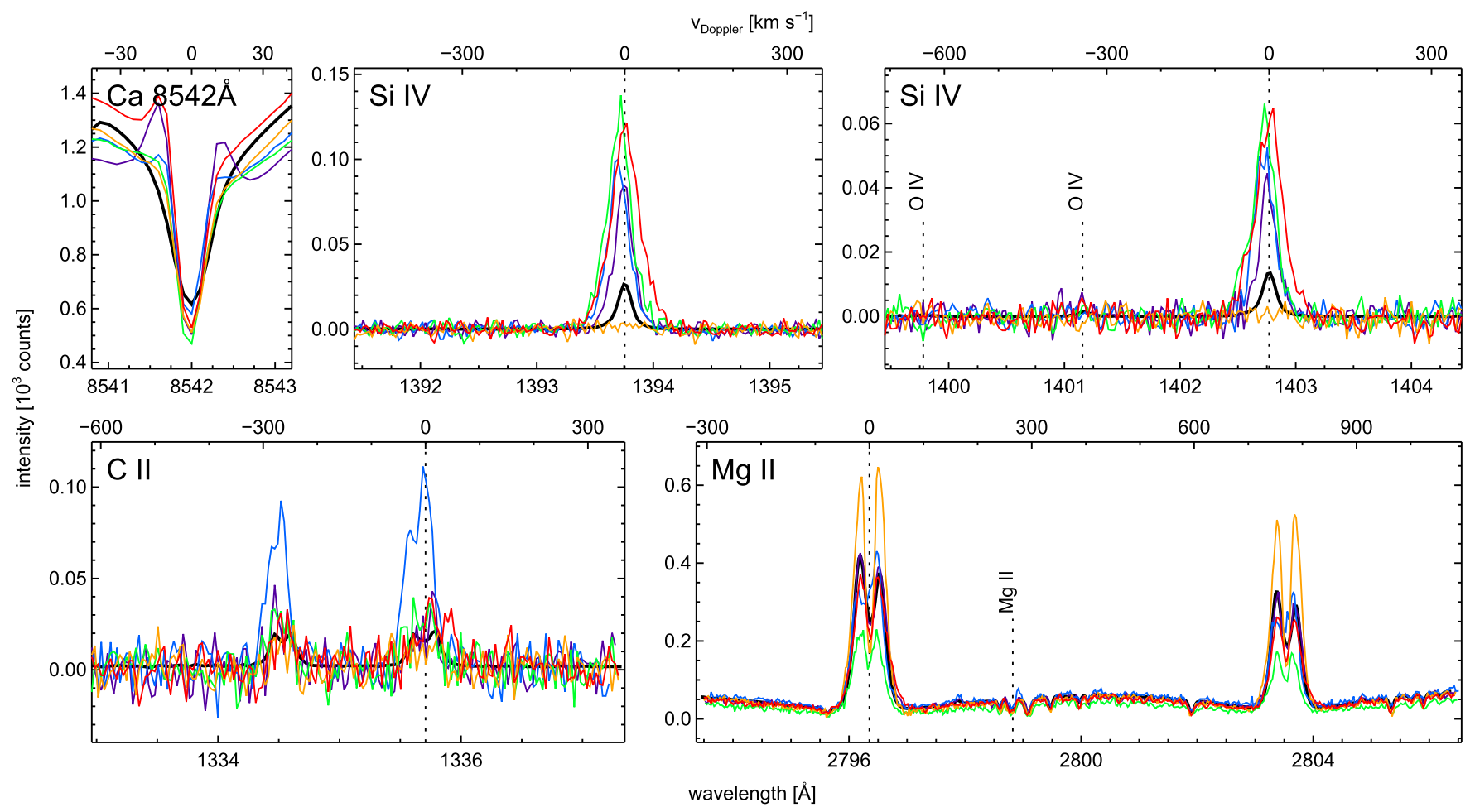

Figure 5. Sample spectra for five PMJs highlighted with the same color-coding in the second lower panel of Figure 1. For each, the IRIS spectra have been obtained at the same location (plus markers, +), while the Ca II $8542 \AA$ stem from the base of the PMJ (cross markers, $\times$ ). The thick solid black line is the average over the blue box in Figure 1 (for the IRIS spectra) or the full SST field of view (for the Ca II $8542 \AA$ line).

$\mathrm{Mg}$ II $\mathrm{h} \& \mathrm{k}$ lines as well as the $\mathrm{Mg}$ II triplet lines at $2798.82 \AA$ (indicated by the labeled vertical dashed line in the lower right panel). Comparison with the images and spectra in $\mathrm{Mg}$ II $\mathrm{k}$, Ca II $8542 \AA$, and $\mathrm{H} \alpha$ suggests this is due to absorption by an overlying fibril that has been blueshifted by the inverse Evershed effect.

As Figures 2 and 3 already suggest, the Si IV or $\mathrm{C}_{\text {II }}$ signal is generally not co-located with the $\mathrm{Mg}$ II $\mathrm{k}$ signal. Hence, it is not surprising that the response in $\mathrm{Mg}$ II $\mathrm{h} \& \mathrm{k}$ is generally weak (if at all present) in most samplings shown in Figure 5. Indeed, the response in the $\mathrm{Si} I V$ and $\mathrm{Mg}$ II $\mathrm{k}$ lines appears to be anticorrelated at these sampling locations: the stronger the $\mathrm{Si}$ IV (increasing for violet, blue, red, to green), the weaker the $\mathrm{Mg}$ II $\mathrm{h} \& \mathrm{k}$. Conversely, the orange sampling, which has been selected specifically to show the PMJ $\mathrm{Mg}_{\text {II }} \mathrm{h} \& \mathrm{k}$ response, shows nothing but noise at the rest wavelengths of both Si IV lines. Compared to the quiescent profile, both $\mathrm{Mg}_{\text {II }}$ lines increase in intensity only in the $\mathrm{k}_{2 V / R}$ and $\mathrm{h}_{2 V / R}$ peaks; the $\mathrm{k}_{3} / \mathrm{h}_{3}$ cores appear in fact darker at this sampling location than the average profile. Although both lines get broadened, the effect is not as strong as for Si IV.

\section{DISCUSSION AND CONCLUSIONS}

We have presented a study of the transition region signature of PMJs in coordinated observations of a sunspot by the SST and IRIS on 2014 September 5-6. Our key finding is the progressive visibility of PMJs in chromospheric and transition region diagnostics along their extent: from base to top the jets are mainly visible in $\mathrm{Ca}$ II $\mathrm{H}, \mathrm{Mg}_{\text {II }} \mathrm{k}$, and $\mathrm{Si}$ IV/C II. These results support the suggestion by Katsukawa et al. (2007) that PMJs may have a transition region component.
The time delay between visibility in these diagnostics is close to negligible, differing at most by about $10 \mathrm{~s}$. Given the characteristic formation temperatures of these lines $\left(10^{4.0} \mathrm{~K}\right.$ for $\mathrm{Mg}_{\text {II }} \mathrm{k}, 10^{4.3} \mathrm{~K}$ for $\mathrm{C}_{\mathrm{II}}$, and $10^{4.8} \mathrm{~K}$ for $\mathrm{Si}$ IV), as well as the spatial offsets along the jet direction (typically at least $500-1000 \mathrm{~km}$ ), there is likely to be an intrinsic time delay in the diagnostic visibility, however, the current cadence does not allow disentangling these conclusively.

The PMJ-related signal in $\mathrm{Mg}$ II $\mathrm{k}$ and $\mathrm{Si}$ IV/C II generally follows the extension set by the $\mathrm{Ca}$ II $\mathrm{H}$ jet; of all 147 cases considered, only two showed some curvature of their path. Such behavior was already reported by Jurčák \& Katsukawa (2008), who found a small angle between the initial and final orientation of a PMJ in consecutive frames. Even though many PMJs appear slender throughout, we find that some jets may broaden up to about $500 \mathrm{~km}$ toward the top. The maximum extents (i.e., combining $\mathrm{Ca}$ II $\mathrm{H}, \mathrm{Mg}$ II $\mathrm{k}$, and $\mathrm{Si}$ IV signal) that we find are of the order of the values reported for $\mathrm{Ca}$ II $\mathrm{H}$ alone by Katsukawa et al. (2007), while the Ca II $\mathrm{H}$ lengths in our samples appear to be much shorter (about 0.5-1 Mm, comparable to the values found for Ca II $8542 \AA$ PMJs by Drews 2014).

Recently, Tian et al. (2014) reported on IRIS observations of bright dots in sunspots, the majority of which were observed in the penumbrae. The dot-like brightenings in their Si Iv slit-jaw images appear to have properties similar to the PMJs in our data: they have similar small round shapes, lifetimes on the order of a minute, and those that show proper motion have comparable speeds of $10-40 \mathrm{~km} \mathrm{~s}^{-1}$ (our PMJs fall on the lower end of that range). The IRIS response is also comparable in the enhancement of the Si IV $1402.77 \AA$ and $C_{\text {II }}$ $1334.53 \AA$ lines, the weak $\mathrm{Mg}$ II $\mathrm{k} 2796.35 \AA$ response, and the absence of significant continuum enhancement and $\mathrm{O}$ IV 
$1401.16 \AA$ A signature. Tian and co-workers suggested that some of these dots could be related to PMJs, given the weak but present $\mathrm{Mg}$ II $\mathrm{k}$ signal, but that many were likely related to energy releases at loop footpoints (in particular those with counterpart signature in the coronal channels of the Solar Dynamics Observatory's Atmospheric Imaging Assembly, Lemen et al. 2012). Considering our findings, we speculate that indeed a large number of their moving bright dots may have been PMJ tops.

The Ca II $8542 \AA$ spectra from the base of the PMJs generally show a blue-over-red asymmetry peaking at some $15 \mathrm{~km} \mathrm{~s}^{-1}$, in accordance with literature values (Reardon et al. 2013; Drews 2014). The interpretation of such a profile is not straightforward, as was already pointed out by Reardon et al. (2013). The peak may represent an actual Doppler component at that velocity, but could also merely be a result of the overlying canopy as, for instance, for Ellerman bombs (Watanabe et al. 2011; Vissers et al. 2013). In at least one example (i.e., the blue profiles), the latter appears to be the case. On the other hand, a few examples of a similar, cotemporal blueshift velocity in both $\mathrm{Si}$ Iv lines and the Ca II $8542 \AA$ line are found, suggesting a persistent Doppler shift throughout the PMJ. Whether these are representative, however, is difficult to say without a larger statistical sample of IRIS spectra. Although the Ca II $8542 \AA$ PMJ profiles are comparable to those of Ellerman bombs, the enhancements in the $\mathrm{Si}$ IV, $\mathrm{C}_{\text {II, }}$ and $\mathrm{Mg}$ II $\mathrm{h} \& \mathrm{k}$ lines are no stronger than the weakest IRIS Ellerman bomb profiles reported in Vissers et al. (2015); more often than not they are much weaker than those.

In conclusion, PMJs have a clear corresponding signature in the IRIS upper chromosphere and transition region diagnostics. The progressive visibility in $\mathrm{Mg}$ II $\mathrm{k}$ and $\mathrm{Si}$ IV/C $\mathrm{C}$ II following the initial jet in $\mathrm{Ca}$ II $\mathrm{H}$ and $\mathrm{Ca}$ II $8542 \AA$, combined with the enhanced and broadened Si Iv lines, suggest heating of plasma to transition region temperatures as it extends upward (while also slightly expanding, likely following the expanding canopy fields). A natural next step would be to expand this study statistically, preferably by sampling a larger part of the penumbra with the IRIS raster than was done in these data sets, as well as doing so at a higher cadence to better disentangle their time evolution.
Our research has been funded by the Norwegian Research Council and by the ERC under the European Union's Seventh Framework Programme (FP7/2007-2013)/ ERC grant agreement nr. 291058. IRIS is a NASA small explorer mission developed and operated by LMSAL with mission operations executed at NASA Ames Research Center and major contributions to downlink communications funded by the Norwegian Space Center through an ESA PRODEX contract. The SST is operated on the island of La Palma by the Institute for Solar Physics of Stockholm University in the Spanish Observatorio del Roque de los Muchachos of the Instituto de Astrofísica de Canarias.

\section{REFERENCES}

de la Cruz Rodríguez, J., Löfdahl, M. G., Sütterlin, P., Hillberg, T., \& Rouppe van der Voort, L. 2015, A\&A, 573, A40

De Pontieu, B., Title, A. M., Lemen, J. R., et al. 2014, SoPh, 289, 2733

Drews, A. 2014, Master thesis, Univ. Oslo, available from: http://urn.nb.no/ URN:NBN:no-46622

Jurčák, J., \& Katsukawa, Y. 2008, A\&A, 488, L33

Jurčák, J., \& Katsukawa, Y. 2010, A\&A, 524, A21

Katsukawa, Y., Berger, T. E., Ichimoto, K., et al. 2007, Sci, 318, 1594

Katsukawa, Y., \& Jurčák, J. 2010, A\&A, 524, A20

Kosugi, T., Matsuzaki, K., Sakao, T., et al. 2007, SoPh, 243, 3

Langhans, K., Scharmer, G. B., Kiselman, D., Löfdahl, M. G., \& Berger, T. E. 2005, A\&A, 436, 1087

Lemen, J. R., Title, A. M., Akin, D. J., et al. 2012, SoPh, 275, 17

Peter, H., Tian, H., Curdt, W., et al. 2014, Sci, 346, 1255726

Reardon, K., Tritschler, A., \& Katsukawa, Y. 2013, ApJ, 779, 143

Scharmer, G. B., Bjelksjo, K., Korhonen, T. K., Lindberg, B., \& Petterson, B. 2003, Proc. SPIE, 4853, 341

Scharmer, G. B., Narayan, G., Hillberg, T., et al. 2008, ApJL, 689, L69

Shine, R. A., Title, A. M., Tarbell, T. D., et al. 1994, ApJ, 430, 413

Tian, H., Kleint, L., Peter, H., et al. 2014, ApJL, 790, L29

Tsuneta, S., Ichimoto, K., Katsukawa, Y., et al. 2008, SoPh, 249, 167

van Noort, M., Rouppe van der Voort, L., \& Löfdahl, M. G. 2005, SoPh, 228, 191

Vissers, G., \& Rouppe van der Voort, L. 2012, ApJ, 750, 22

Vissers, G. J. M., Rouppe van der Voort, L. H. M., \& Rutten, R. J. 2013, ApJ, 774,32

Vissers, G. J. M., Rouppe van der Voort, L. H. M., Rutten, R. J., Carlsson, M., \& de Pontieu, B. 2015, ApJ, in press (arXiv:1507.00435)

Watanabe, H., Vissers, G., Kitai, R., Rouppe van der Voort, L., \& Rutten, R. J. 2011, ApJ, 736, 71 